weijen Foritmeifter Senfelt in gerabezu muiftergültiger $\mathfrak{B e i f e}$ unter zum $\mathfrak{T}$ eil fdwierigen Berbältniffen mit bejtem criolge burägefübrt bat. überoies finbet fich hier bereite ein moderner Grunbjab in bie \$raxis übertragen, indem es $\mathfrak{u}$. a. beipt: "\$Bevorzutgt wirb ftets ber 2 Ingriff pon Morben ber."

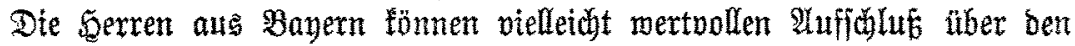

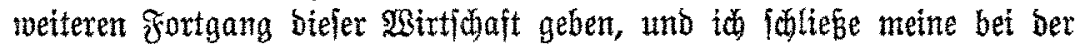
Zülle bes Stoffs mehr auszug weife gegebemen Darlegungen mit bem

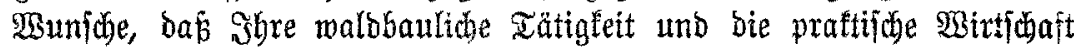
überbaupt §hnen biejelbe innere Befriebigung und Freude bereiten möge, wie mir bie jeit 10 Sabren geübte grunoföb̨lide Betätigung ber natür= lichen \$erjüngung nach ber Anleitung unjerer grob́en Rebrmeifter (Gayer und Guttoegagen, für beren Soeen neuerbings Seinrti Mayr uno Ehriftof $\mathfrak{B a g n e r}$ als witroige Tadjolger eifrig und boffentitio exfolgreid Sdule machen, weldie ber jüngfte Snbaber bes Gaver'fohen

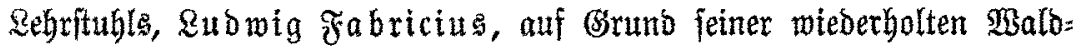
baultubien bier unb vor allem in jeinem engeren Sgeimatlande, boffen wir, mit voller überzeugung vertreter, zu Put und Frommen ber \$ratgi fortbitDen und meiter verfümber miro.

\title{
II. Hitteilumgen.
}

\section{Beriḑt über den waldbanfurs in Sangenbrand im September 1913 .}

Dberföriter Dr. \&bergard=\&angenbrand, Der \&eiter uno Beranitalter bes Surjes, begrînte am 2 benb bes 1 . September in Salmbach bie zablreid eridienenen Teilnebmer aus Bayern, Baben, SBürttemberg uno Follano und entrotdelte ben (orrunbgebanfen ber ganzen Beranitaltung:

Föroerung uno Bertiefung ber miffenidgaftliden Erfenntnie burdy

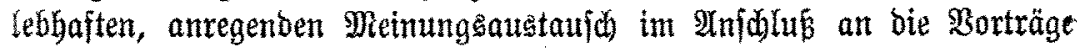
verbunben mit einer grünbliçen Beiprectung ber fich auf ben (Exfurfitoner axgebenden Fragen uno Bebenten am einzelnen Dbjette wäbrenob bec Maldbegangs.

Sobann wurben an ber Sand bes jebem Teilnehmer mehrere Tage vor Beginn bes Surjes überjandten ₹̧ührers eingebende Erläuterungent

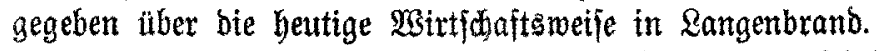

Siernad Gat ber Foritbezir Eangenbrand eine Flädhengröbe von 2242 ha, gegliebert in 81 5iebszïge, 9 Diftrilte, 154 2lbteilungen mit 444 Unterabteilungen, nebit 687 ba Gemeindewaldungen. 


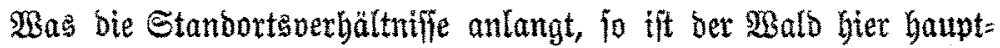

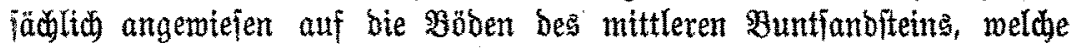
mannigfach Drtfein in verfdiesener Mädtigteit und Tiefe enthalten. Die frubtbaren $\mathfrak{B a b e n}$ bes oberen Buntjanditeing itno mux auf tletneren

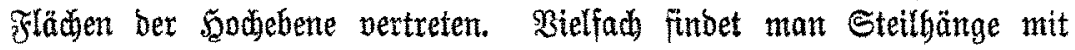
Felsüberlagerung.

Die Sieberichlagsmenge beträgt bei einer Meereshähe von 400 bis

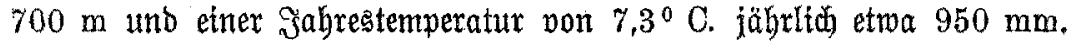
Sturm= und Froitgefahr fpielen leine nennensmerte Rolle.

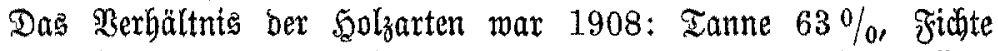
$14 \%$, Riefer $17 \%$, Laubholz (meift Butche) $6 \%$. Das altergflaflen= verbältnts ftellt fich folgentermab̌en bar:

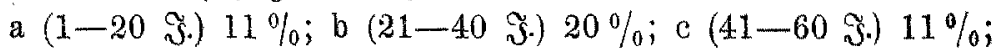
d $(61-80 \Im$.) $13 \%$; e $(81-100 \Im$.) $29 \%$ unb f (über $100 \Im$.) $16 \%$.

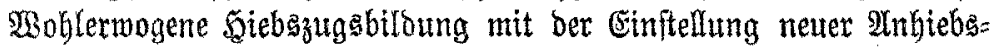
Inien jowie bie Serabjebung Der Umtriebszeit pon 120 auf 100 Sabre

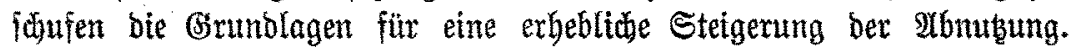
Diejelbe beträgt $1909 / 18 \quad 18400 \mathrm{fm}$ Ento= uno $3300 \mathrm{fm}$ Bormub̨ung ober pro Seftar $9,7 \mathrm{fm}$; gegenüber $1888 / 97$ mit $6000 \mathrm{fm}$ Szaupt= uno $3000 \mathrm{fm}$ Rornub̧ung, ingefamt $9000 \mathrm{fm}$ ober $4 \mathrm{fm}$ pro $\mathfrak{S e f t a r}$ $1898 / 1908: 9000+2000=11000$ ober $4,9 \mathrm{fm}$ pro Seftar -.

Dieje 3ablen gewinnen an Bebeutung, went wir ihnen ben jähr= lidhen Rulturaufwano gegenüberitellen. Derjelbe beläuft ftă: 1899-1908 auf 2284 th im Durafidenitt unb etwa 1 .h auf 1 Seftar $1909-1913$ " 1561 " " " 0,69 " 1 "

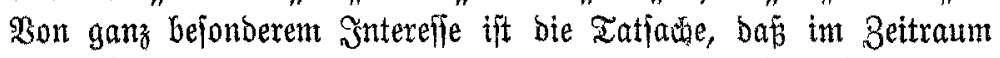
1905-13 bei einer 5eauptnutbung von 135000 fm auf rund 247 ha,

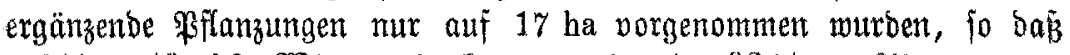

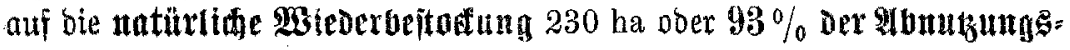
fläd) entfallen. Sin biejen Bablen fpridgt fich bie Berwirtichung bes

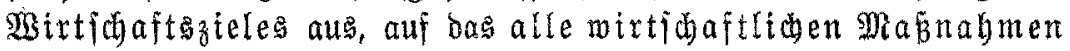

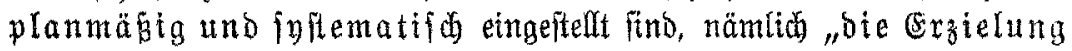
ciner ftanbortagemäran geficherten beitanbesmifoung mit Begüntigung ber wertoolliten Solzart uno unter Berite

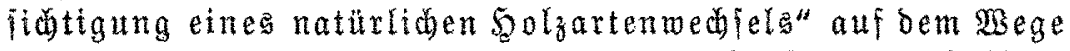

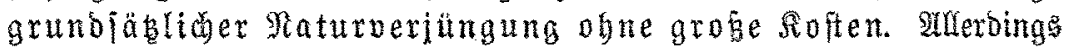
milfen wir befien eingebent fein, baj bie gegebenen Berbälniffe bes

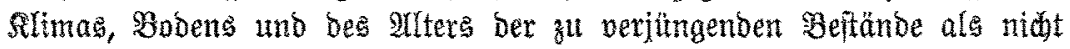
ungünftige anzulpredien fint. Dabei banbelte es fich um bie Snangriff=

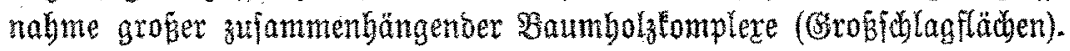


Mls eine befonbers widtige Morbebingung für einen fiberers

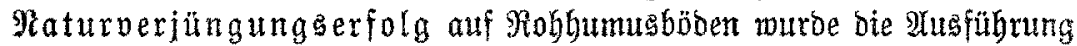

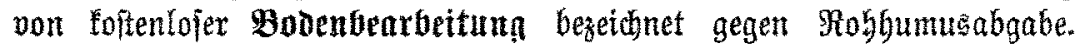
Der Bobent wirs plabzeife in $1 \frac{1}{2}$ qm $B$ Berbanb bearbeitet, mobei berjelbe

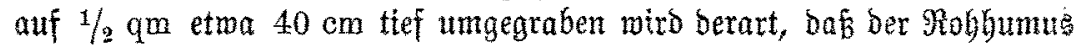
nach unten unb ber mineralitide Boben nad oben gefehrt mirb bean. eine leichte Bermengung beiber ftattfinbet. Bei geringer Mäudigfeit ber Streubecte miro bie Bobenbearbeitung im Iaglohn bejorgt. Der Boben= uberang wiro nun, foweit er aus Seibe unb Sauermoos befteht, gant entfernt; foweit er aus Seibelbeeren und 2yitmonfen fiti zujammenjest, auf $1 \mathrm{~m}$ treiten Streifen unter Belajing oer Bobended auf $1 \mathrm{~m}$ breiten 3wifchenftreifen. Sn bieier $\mathfrak{B e i f e}$ wurben $1907-13 \quad 115 \mathrm{ha}$, Giervon Eoftenlos 105 ha, für bie Beriünung vorbereitet. Sine bem $\mathfrak{B} e r=$ jüngunggfaum voraugreifende $\mathfrak{B}$ orblenberung ber Beftände wirb auf

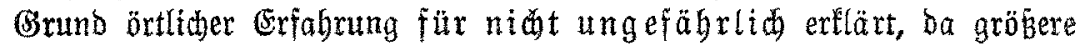

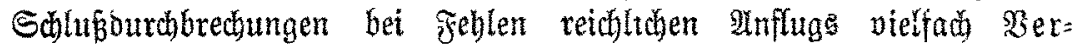
untrautung und Berwilberung des Bobens bemirlen.

In biefe allgemeinen crörterungen follob fid ber erfte Sortrag von

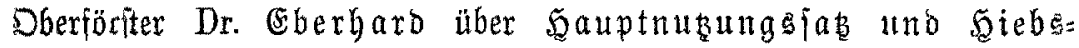
fortidritt, welder bejonders veroffentlidt wirb.

Sn ber bem Bortrage folgenben Debatte murbe zunädjt bie Frage ber faumweifen Berjügung ber leidt= unb fowerjamigen

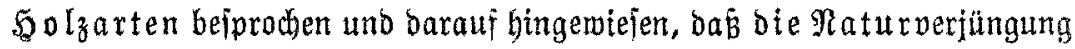

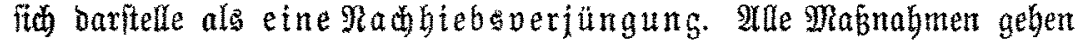
barauf aus, in allen hiebsreifen Beftänden ohne ftärkere Eingriffe

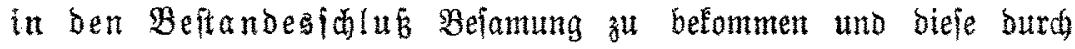
mäbige Durchjoritungen, foweit Untrautgejabr zu bejorgen if, lebentäb̆g zu erbalten. Die ftarfen Durdforitungsgrabe baben fith unter ben \&angen=

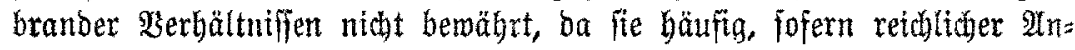
flug ausbleibt, zu Bobenverangerung führen. Alukerbem fei bie Boben= bearbeitung ein febr wertoolles Silfsinittel für bas fidere Gelingen ber

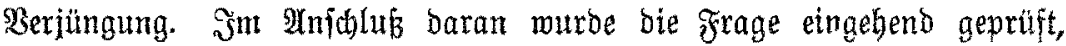
wie weit man burbfontitid jabrlid ben (serablatm vor: fohieben lönne bei Unterftellung ber Erbebung ber vollen natbyaltigen Nutung und bei Anmenoung einer walbpfleglichen Ernte. Es wurbe

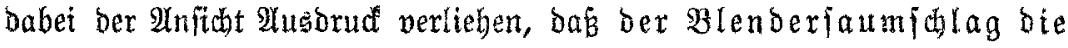
zur Etaterfüllung nötige Saumzabl wobl zul liefern vermöge bei einem 5iebsfortfidritt pon $15-20 \mathrm{~m}$. So foller in bem bis

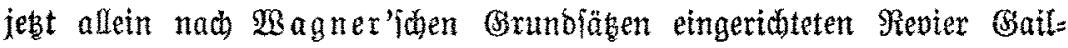

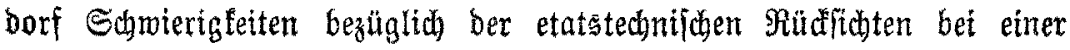




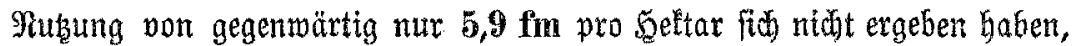

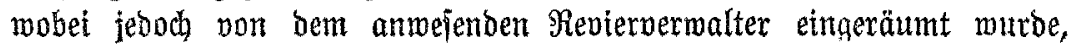

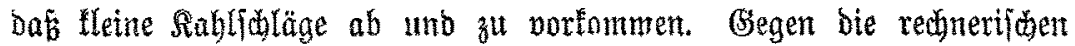

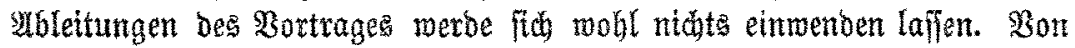
anderer Seite muroe bann now auf bie Moghlidfeit hingewiệen, fith mit Durdhtieben zu belfen.

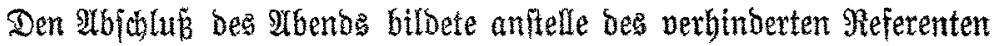
ein Sortrag von Dberförfter Dr. Eberbarb über bie Wirtfogft in Rangenbranb in ben lebten Gunbert Sabren unb ibre sebren.

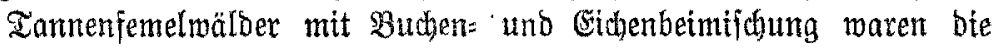
Balbbeftochung zu Anfang bes 19. Sabrbumberts. Mit grosem Eifer wurbe bie Raturverjüngung ber Tonne betrieben gegentuber ber fith von

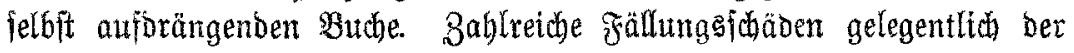

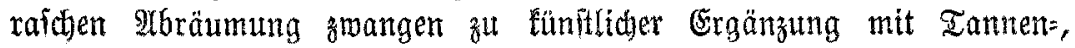
Sidten, Riejern unb Birfenjauten, wozu balo \$flanzungen famen. Man

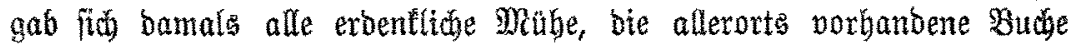
aus bem Balbe zu verorängen, um ber Tamne zu bejonberem Gebeiben zu verhelfen. Seute fteht uns das Ergebnts biejes Bernidutungstampfes

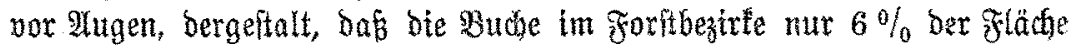

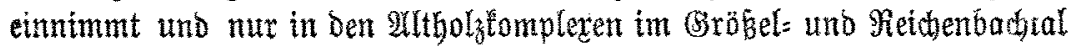
in nennenswerter Form vertreten it. Sin itbrigen Tetle bes Reviers treffen wir bin und wieber bie Buche zrifjen= und unterfitänoig. Bald folgte bie 8eit ausfchlięlicer Sumitneriungung, zumal auch bie Ratur=

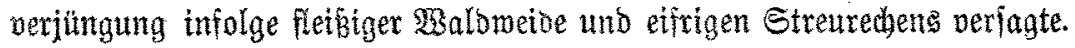
Die Mirtiobafisregeln ber jethjiger Sabre förteben zwar von neutem Matur=

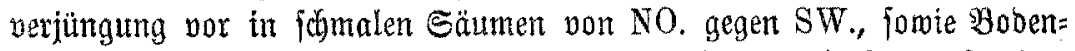

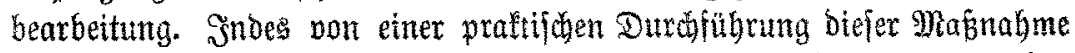
im groben ift nie die Rebe. Die Sorausfetumgen biez̧ waren eben nod nidgt gegeben. (Erit in jüngter Beit wurbe auf bie $\mathfrak{M a t u r =}$

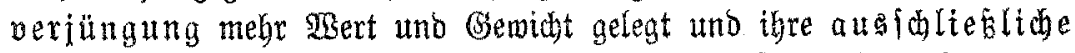

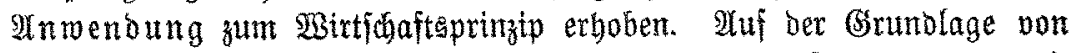
(B)ayer's Sdirmbejamung in Saumlalägen, in Bufammenbange mit

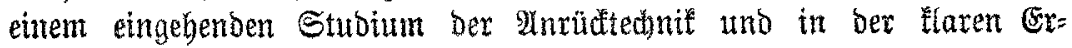

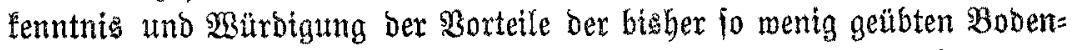
bearbeitung bildete fich bas Berfabren bes Mebriaumbetriebes aus, beffen erfolgreide Duroffübrung an bie Reifungafäbigkett uno bie Spann=

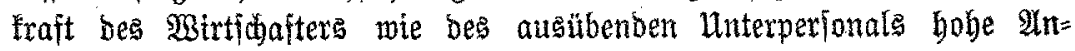
forberungen fiellt.

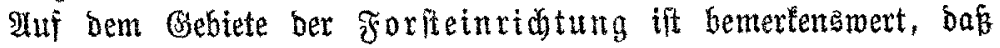
bie Bejamtorbholzntubung im Sabre 1809 für faft 60 gabre im voraus 


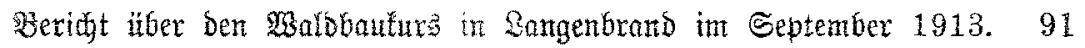

richtig bemefien murbe. Beponbere Fortidritte und Errungenidaften

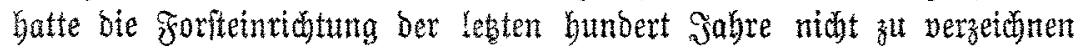

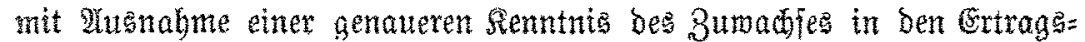
tafeln. Erft im leten Sabrzebnt gewannen bann biejenigen Befitrebungent

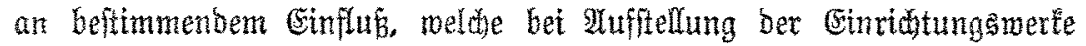

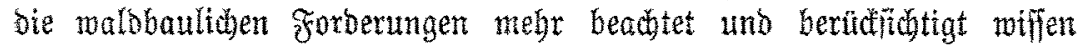
wolten uno die Ertragsfeftlegutng uno Erhebung an cinzelnen Drt jenent anzupafien fucơten.

Orbnen wir bas betonomifo Bringip bem natürliden unter, bann ift ber böbfte finanzidele Erfolg fir immer geftchert, fo meinte Der Bortragenbe. Aller unb jeder Fortideritt ift gefnüpt an bie Beoingung einer wejentlid freien Bairtidaft. Das Mafacbenoe

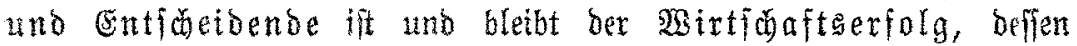
ridutige Darftellung Doerföriter Dr. Cerbard mit als 3 weed unb (Srumb= lage ber periobilcten Forfteinrichtung bezeichnete.

Der erfte $\mathfrak{S}_{\text {alobegang am }}^{*}$ folgenben Tag fübrte in Diftrift III und VI des Reviers Rangenbrand. Fn der Hbteilung Fans= walb (III, 3), etnem 99 jäfrigen Zamnenbaumbolz I. - II. Bonität, wurben bie eriten von Roro nach Sü veriaufenben Säume mit oft=meftlider Siebridutung vorgezeigt. Dieje ausnahmstoije Saumridutung ift bedingt burd bie von Form unb aage ber 2 bteilung abhängige

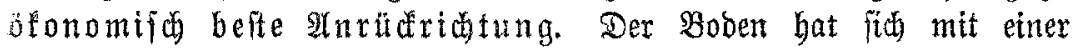

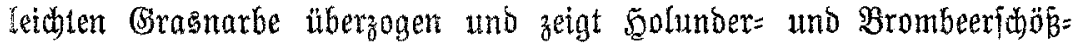
linge, bte zu langiamem Ştebefortichritt ofgne Borblenberung zwingent. (Senügento 2 (njamung if überall fu finden.

Ybteilung 5 (Erztoajd), ein Gumbertiäbriger Tamenteitond, ift ourch

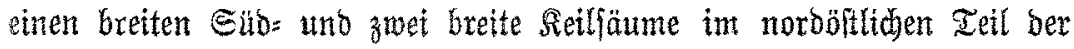
Wbteilung in \$erjüngung genommen. Snfolge ber räumigen Stellung

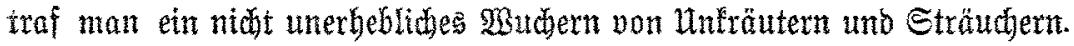

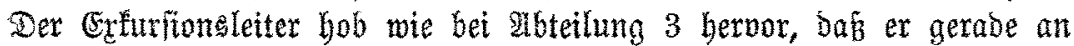
biejem Drte, welder früber gan unfrautfei gewejen jei, bas fo wichtige langiame sorgeben gelernt babe. Ein idimaler, langer Setlfaum im norböftliden Teil ber Abteilung geigte eîn vielveripredenbes Bilb. Dem= entipredieno bat fich jenes Beriungungsuerfabren entridfelt, wie es Dber=

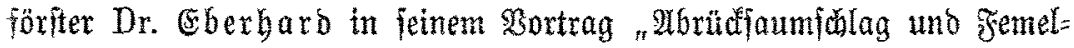
Fislagbetrieb" eingebeno begondelt.

Wetterbin trat in einem bunbertjährigen Tannenbeitano III. Bontät (VI, 5) beutlici) vor צugen, wieviel leichter fich bie Maturberjüngung polfziebt auf wentger frubtbaren Böben im Bergleid mit guten Stand= 


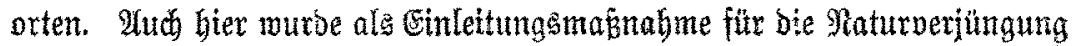
bie Sodenbearbeitung empfoblett. Die Mbteilung wiro von zwei Reti= fäumen und einent Gerabfaum won Süben ber angegriffen. Boden= bearbeitung und ber burch bie Dreizahl ber Säume ermögliöte lang: jame Siebsfortichritt bringen in bem fteturauben Gelände einen an= gemeffetren Berjüngungserfolg. arferoings barf nicht verididegen werben,

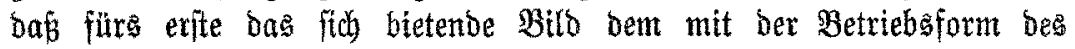
Seilfaums nidst vertrauten Befhauer wobl etwas uniberfichtlich eridien, fomie ba bie ftellenweije vorbandene Bobendecte von நetbelbeeren einen wenig günftigen Cinbrud berworrief. axllein bei näberem 3 ufehen ent= becte man itberall eine bimreidjenbe $3 \mathfrak{a h l}$ von jungen \$flänłlingen, wenn: gleich béejelben hin unb wieber $1 \mathrm{~m}$ und meghr voneinander entjernt waren. Ŝn anberen 2ybteilungen fonnte aud bie Beobaditung gemadt.

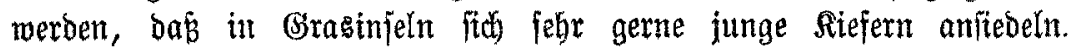
Durdaus volfommen war bie Beriüngung in ben bearbeiteten söbern, in welchen fie ein belonders gutes 2 lusieben zeigte. Ein in

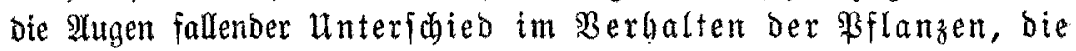

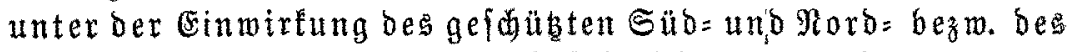
Dft = uno weftfaums ftanden, ift jebr ibwer feftzuftellen, wem. man bie Beftandestänber abgeht unb obne Boreingenonmenheit $\mathfrak{B e r}=$

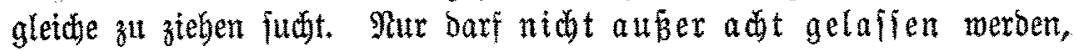
bas bie Berjüngung von Süben wie von Diten ber grandjąlidi nibt auf offener Saumfläbe, fonberm im gelobuten altbolzrano bes Retlfaums vor fich geht. Răb ber Freiftellung wawien bie jungen \$flanzen fräftig unb lafien burchaug Kein Rümmern erfennen. Şaben fie bod) in Dem beifen Sommer 1911 dem Sonnenbrand getrobet, ohne irgenoweldhen Shaden zu nehmen.

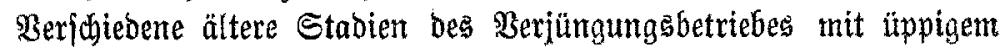
Madistum traf man in VI, 6, 7, 8, welde ein flares, anjoualidjes Billo geben von bem langiam und ftetig vorgebenden, jebe einzelne $\mathfrak{B e}$ :

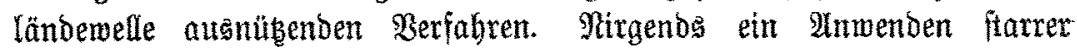
Grunbjäbe, fondern ftete ein 2 npaffen ber Maṕregeln und Mirtịdafta grumbiäke an bie Bebürfniffe bes einzelnen Drts. Ränge ber Wege feben

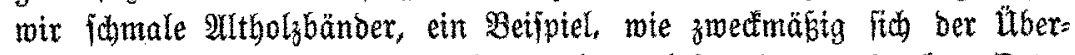
baltbetrieb an bie Beftandestänoer verlegen läft. Hn vereinzelten Drten gewähren hod ragende, fhlanfe Sŭwarzwaldfiefern bem Iuge etne lteb: lide, reizpolle albmechjelung.

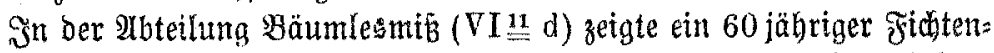
beftand mit etwas Riefern= unt Tannenbeimifdutug auj Rlebjandboben, bas Produft Iangjäbriger Sulturveriuche aus ber Rablichlagperiobe un 


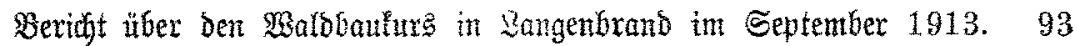

bie Mitte beg vorigen Sabrhunberts, bie erfiten $\mathfrak{A}$ nfänge von Tannen= anflug. Weutzutage gibt uns bie Matur jelbft Fingerzeige, wie fie ben Bejtand befandelt wiffen will (Solzartenwedbjel) ohne 3 ufubr won Mineral= bünex tho obne Einbringen von Budben.

Mad einet furzen Streffe Segs burdi bie Gemeinbewalbungen von Salmbach erreidte man balb bas freunditue Salmbach, wo nad einer längeren Baufe Sandeggeologe Dr. Regelmann = Stuttgart mit bem eriten

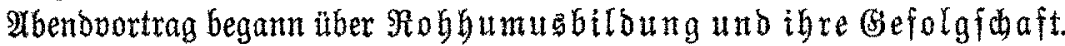

2luggebend von ber geologifiden Entmidtung des Schwarzwalbes fobilderte ber Reoner bie Bebingungen ber Rohbumug= uno Bleidfanbbiloung jowie ber Drtiteinablagerung und bie Eigenjuaften berartiger Böben. Unjere Beahtung verbient bie Feft= ftellung, bå̉ auf Drtfteinböbent bei nidit zu geringer Mäutigleit ber

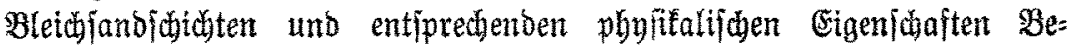
ftänbe mit gutem $\mathfrak{B a c h s t u m ~ a n g e t r o f f e n ~ w e r b e n . ~ S ̧ a ~ j o g a r ~ l o ̉ n e ~ u n t e r ~}$

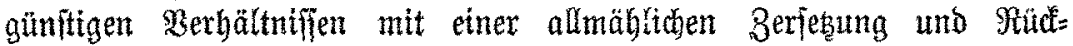
billoung ber Drtíteinfouidyten gerechnet werben. Das Ceterum censeo

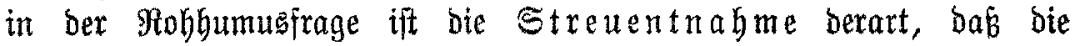

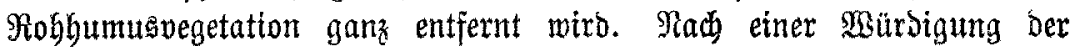
zwectuääigen Bebandung biefer Bobentrantheiten wutben bie waraltes riftichen Mertmale uno Rennzetaen ber Rlebjanobiloung aufgezăhlt uno bie noch ungetlärte Frage ifrer Befämplung geftreift mit ber Alfforberung an bie forftliche \$raxie zu elfriger Mitarbett an biefem fobmierigen Probleme.

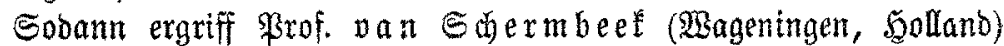
bas $\mathfrak{B o r t}$ zu einer Fede über Affimilation und $\mathfrak{B}$ uds ber Bäume, insbejondere Energieveridmendung beim Rabl= jălagbetriebe.

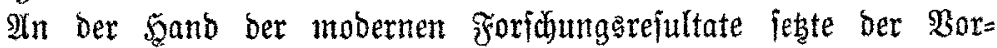

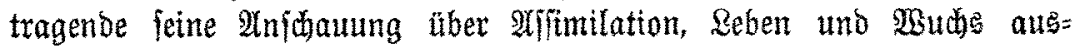

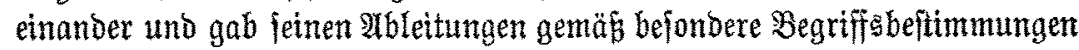
über biefe Raturvorgänge. Des weiteren entwidelte er als Rejultat

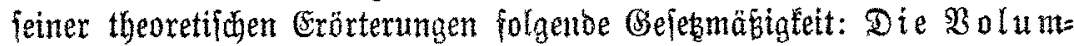
mebrung, bie Bellmandoeritärang, bie Rernbiloung bes Baumeserfolgt immer proportional bem ber Baumlörper an etner beftmmten Stelle zu leiften hat.

Derartige Semmungen finb Gervorgerufen einerjetts burd Drut= wirfungen, benen ber Baumföeper von auben her unterliegt, zum andern ouro Beftrablungsfợanfungen in längeren ober fürzeren Beiträumen. Photographien von Demonitrationematerial, welde vorgezetgt wurben, 
94 Beridgt itber ben Walbbauture in Rangenbrand im September 1915.

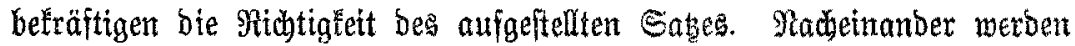
beiprochen: Hyponastie, Epinastie, und ber Duexichnittemule im afl= gemeinent. Šinftchtlie ber Energienerjobendung im Rablichlagbetriebe

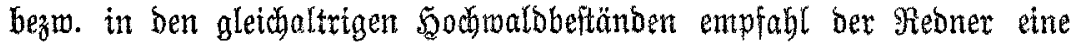

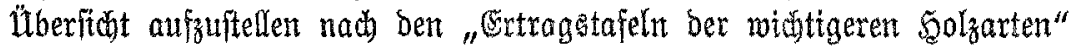
(Meubamm 1913). In biejer 8ujammentitunng wirb vergliden ber laufende 3umadbs pro Seltar mit ber Maffenleiflung pro 100 Stänme:

1. im jüngften in ber Crtragstafel angegebenen 2 lter;

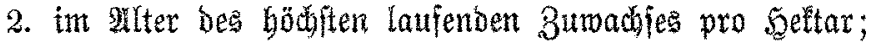

3. im Ulter ber höbiten Bolumleiftung bes Snbivibutms.

Seht man nun bas Maximum bes laufenben 3uwadjes pro Seltar gleid 100 tho bezeidonet man ben Energieberluft in biejen geitpunft mit Null, bann foll eine berartige luberitidt ben Energieverluft in gleids= altrigen Sownalbe mit einem Sromenjtod bartun. -

Xm nächiten Morgen brachte ein Mutoomnibus nach einer Befichtigung bes Dittrift IV bes Reviers Langenbrand bie Teilnehmer nad 2 illsbad zur Exturfion in ben gletdnamigen Foritbezint, befien Berwalter Forit= meifter Dref

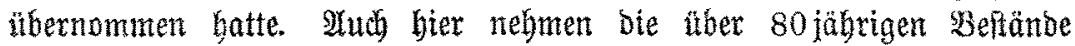
mebr als $49 \%$ ber Fläbe ein unb bieten als grobe, zufammengelagerte

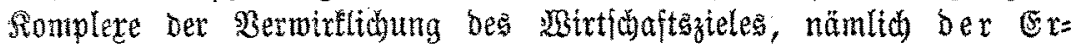
giebung gemijoter Beftände aus Tanne, Bube, Riefer

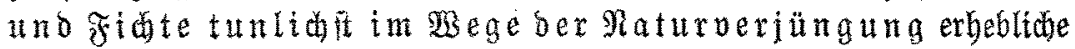

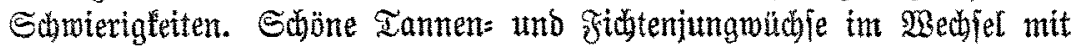
Mltbölzern, naturtibe Serjüngungen uno wohtgelungene Sulturen auf Grobfläacen erfreuten bas aluge. Bor allem mirb ein Billo im Bedädotnis aller baften geblieben fein: eine erfolgreidje natürlide Berjüngung pon Tanne uno Buche unter Sdtrmitano eines etwa 140 jäbrigen 2 Itholzes als 0,7 Tame unb 0,3 Buche ohne Bobenvorberetutng, exfolgreit lebtglid Durb) Inbringen eines Drabtzaunes zum Schu gegen bas zabl= reich vertretene Witlo. Der erfte Machbteb wuroe 4 Sabre nach Ein= leitung ber Berjünung gefithrt. Sm Baufe von $2-3$ Sabren follen meitere ₹räftige Machbiebe womöglid bei Schneebecte folgen.

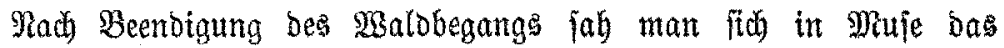
freunblibe Mailbabo an mit feinen retzenben Enztalanlagen. Man jobied

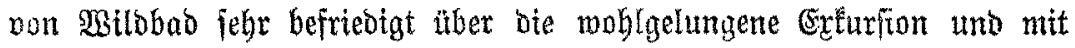
Dant gegen ben liebentwärdigen Füthrer Foritmetiter Dreider.

Wbends in Salmbad fprad \$rof. Dr. Fabrictude Mündyen itber bie Raturverjügungserfabren, ingbefondere bas bayerifde. 


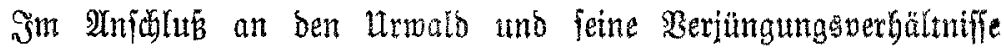
wutroe bas sierben unb Enffteben des alten Femelfwlagbetriebs unferes heltigen Schirmgropidhlage - bargentell, beffen sachtetíe be= fonbers in ber ibm von (S. \&. Sartig verhelenen Form in ber Folgezeit zum Grofkahlfolag fübrten. Gegen biefe Mifachtung ber Ratur wanbte

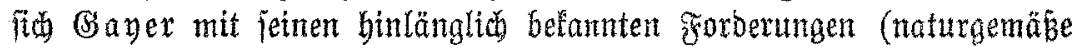
Beftanbesbemirtichaftung mit bejonderer Betontung ber Pflege Der Stand=

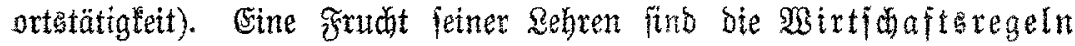
für bie $\mathfrak{B a l b}$ ungen bei fehrbeim a. Donau, weide bas wejen

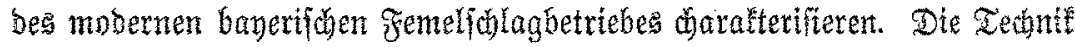

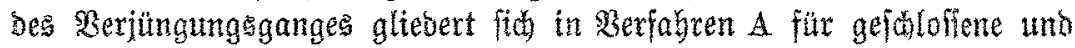

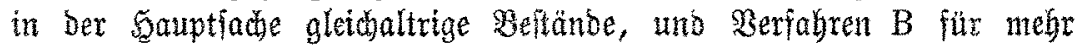
ober wentgex lüđtige uno angleidhaltrige Beftänbe. (Ein näheres cingehen auf bielelben ift mobl überfüfig, oa fre in bem Beridte zut beutd, Forftuerfammlung zo Regengburg 1901 eingeheno enläutert find.)

Dag combinierte Berfahren vereinigt bann bie Borteile bes Femel= Fălagbetriebs mit Sablfäumen von winbentgegengejeb̧ten Setten aủ in Sintereffe einer Befldeunigung bes $\mathfrak{B}$ erjïngungsgangs, wie bie Erfültung

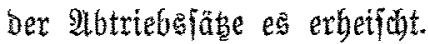

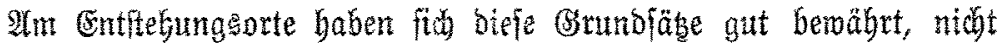
aber an anberen Drten. So mirb benn beutzutage bie Beriünung betrieben auf Dem SBege faumweifer gegen bie Sauptwinbribtung

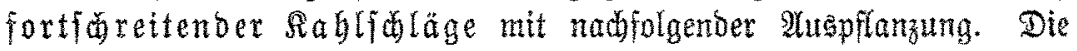

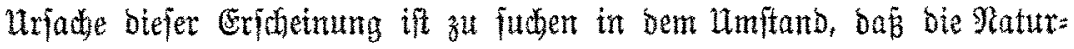

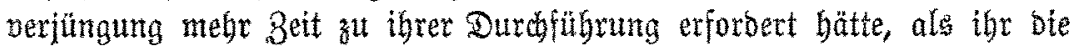

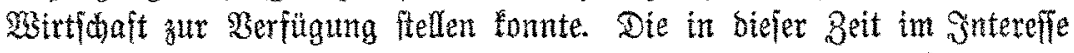

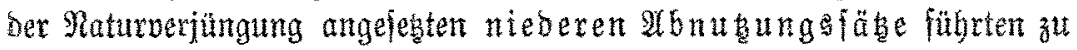

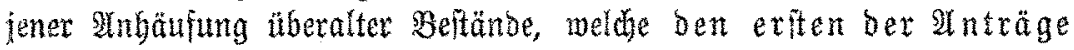
bes Grafen Töring= Jettenbad bernortefen. Der bierauf in ote

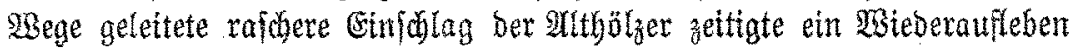
bee (Groplablfolloge.

IUf Grmo biejer Erfahrungen ber lebten Sabrzehnte gebt has all: gemeine Beftreben nunmebr babin, ber Maturyerjünung burd geetgnete

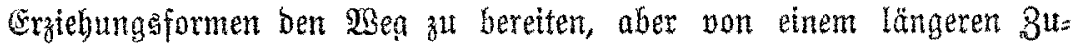
warten auf Raturbefamung abzueben.

Sn Ieţten Bortrage behandelte Dberforifter Dr. Bberharo bos

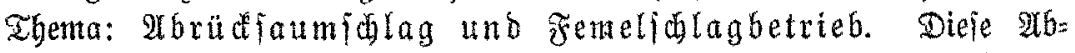
Ganblung fommt ebenfalle in threr Driginalfaffung zut Beroffentlidung in biefer 3eitfdrift. Der vorgerüdten Stunbe wegen fonnte an biejem, wie am vergangenen Wbeno eine Debatte nicht mebr ftattfinden. 
Der 4. September war bent Bejudje ber Difitrifte VII, VIII uno IX bes Forftbegtrte Rangenbranb unb ber Befidtigung ber (Bemeindervaloungen von Grunbach gewiomet.

S̄n VII, 2 unb 3 (f/a) gewann man einen weiteren guten Etnblid in bie hier üblidie Berjüngungsmethode. Bunäbft maren Schwierigletten ber Siebsfolge zu befeitigen. Wbteilung 3 (weillid) mit reiditidem 5 bi 10 jäbrigem Zammenanflug in ber jübliden Sälfte follte nidgt vor 2 (offtlid) genutht werben. Mit Sellfe bes Retljaumes und ber Berjüngung von Süben her wurben beibe Mbtetlungen gleidzeitig in Mngriff genommen. War man feither vielleidt nod nidt ganz ïberzeugt von Der erfolgreidien Durffiübrbarteit Des Berfabrens, fo wurbe mant an Drt und Stelle eines befferen belehrt. Snsbejonbere war das Bild geeignet, Den Bormurf ber Unüberititliblett bes Reilfaumbetriebes zu wiberlegen.

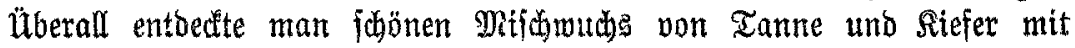

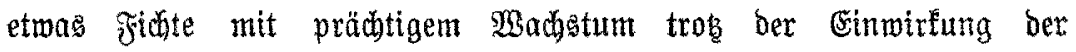
Sitbjonne.

Die Stebsfübrung an einem fteilen Dithange murbe in VIII, 2, einem hunoertjährigen $\mathfrak{B u d j e n a l t h o l z e ~ i n ~} Y$ Inlebnung an bie nebenitebenoen frïberen Sulfiffenichläge in VIII, 1 erläutert.

Sn ben Bemeinbernalbungen pon Grunbadh ift bie Mirtichaft barauf eingeriobtet, bie ïberall vorbanbene natürliche Seriüngung freizutitellen,

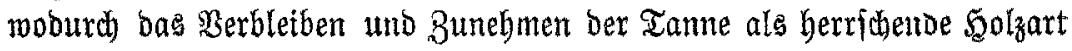

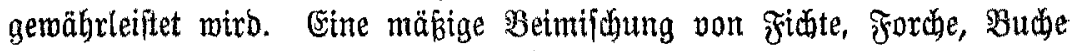
und sidfe foll babei erbalten bleiben, was ebenfalls auf natürlidjem פege erreidt wiro. Die nidft zu grosen abteilungen erleidhtern bie Bermirflidung bes geftedten Biels. Sdone Berjüngungshildoer mit aus: gezeidinetem Bebeiben fanben fith in Menge. Deutlich tritt in bie (ar= fibeinung, wieviel letater und reidlider fich Mijobbeftänoe verjüngen als mehr ober weniger reine $\mathfrak{B}$ eftände. Das angemandte Berfahren ift eine Rombination faummeifer Beriủngung uno borit= und gruppenweifer 9 Birt=

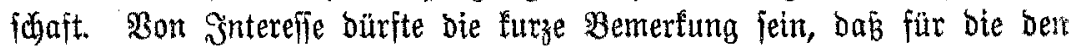
3uwad) um $250 \mathrm{fm}$ überidjreitende Gejantnubung ein Grunditodfionbs befteht; Der Einfachleit balber wirb für bieje jäbrliche Mebrnubung pro

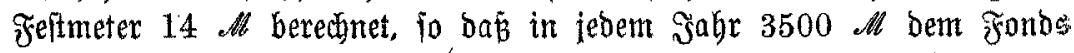

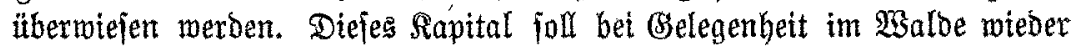

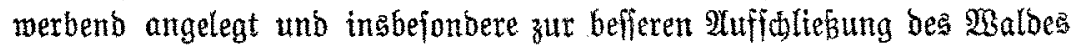
burd $\mathfrak{B e g e}$ verwendet werben.

Mit biejer Exfurfion jojloffen bie Berbanblungen. Die Befriediguma über bie genufreiden $\mathfrak{T a g e}$ war allgentein. Wohl alle Tethebmer febrten in ihre beimat jurüd mit bem Gebanten, mandes Beadteng= und $\mathfrak{P a d}=$ 
Berift über ben Malbbaturas in Sangenbrand im September 1913. 97

abmenswerte gejeben zu hahen unb mit bem (Befühle aufriötigen Danfes gegen ben verbienten Reiter bes Surjes, Dberförfter Dr. Eberbarb.

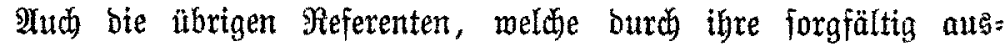
gearbeiteten, flaren Borträge mit zu bem guten Gelingen ber Tagung wejentlich beigetragen haben, dürfen des Danfes igrer 3 uhb̈rer gewín jein.

Die in Sgetbelberg anläblich bes 1. Fortbildungahturjes gewonnenen

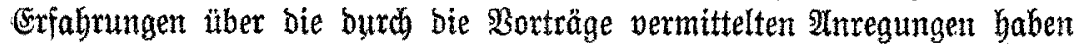
fid) unter hiefitgen Berbältniffen vollauf beftätigt. Sine Zeilnebmerzabl von mur 22 Gerren bürfte zu ben Erfolge mejentlich beigetragen baben. Ferner bat jene in 5ूeibelberg aufgettellte Forberung: "Berfianblungs: gegenftand unb Exfurfion follten mehr aufeinander abgeftimmt werben",

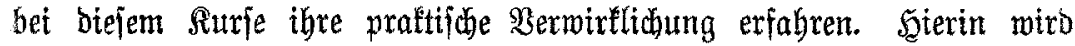
boch wohl ein gewiffer Fortidgritt zu erblicten jein, injofern bie theo= retijohen Forberungen aus ben Borträgen an einzelnen $\mathfrak{B a l b o b j e f t e n ~ v e r = ~}$ anfdaulidgt und ilgre Mitblidfeit am Erfolge gemeffen merben fonnte. Darum wäre es fehr zat begrïben, went nad bem Sorgang bes von Dberföriter Dr. Eberharo ins Seben gerufenen $\mathfrak{B a l b b a u f u r j e s ~ a ̈ h n l i c h e ~}$ Beranftaltungen getroffen müroen, weldye iich in ben fo midtigen Dienit ber Förberung bes praftificen 1 albbaus ftellen. Bejonders werben fiti baz̆u foldbe Reviere eignen, welde eine längere Bewirtidhaftung aufweifen nach einbeitliden Grunbzägen, jelbit menn biefe von ben zurzett herrfhenben IInjobautngen abweiden. Sehen borb heutzutage viele in manchem Ber: fabren eitre 9 Urt von Gebeimmittel in ber Meintng, es ermögliche alle Sthierigteiten obne viel eigene Arbeit uno Anfrengung za bebeben. Da gilt es boch auch für ben Forftmann, fith bes altberäbrten Sabes zu erinnern: Per aspera ad astra.

-g.

\section{Die XIV. Gauptverfammlung des Deutidyen forftwereins in Trier.}

Sin ber lieblichen Moielitaot Triet mit ben itolzen Beugen thret alts ehrwirbigen Bergangengett, beren marfantefte Bertreterin, bie "Porta nigra", in firniger Welfe bie grüne Sulleife ber Berfammlungsteilnehmer gierte, tagle vom 25. mit 28. 2uguit 1913 bie XIV. Sgaptveriammlung

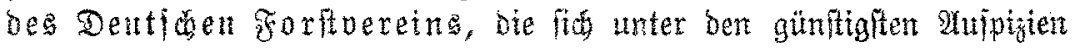
sines fonnigen Syimmels und zablreider Beteiltgung won $4^{1 / 2}$ Sgunbert Teilnehmern in allietts befriebigenter $\mathfrak{B}$ eije vollzog.

Singeleitet war bie feitlidye Tagung burob einen von der Stabs Trier in gaftfretefter Weife gegebenen Begrübunggabenb. Unter ben 